\title{
Calculation of the Masses of All Fundamental Elementary Particles with an Accuracy of Approx. 1\%
}

\author{
Karl Otto Greulich \\ Fritz Lipmann Institute Beutenbergstr. 11 D 07745 Jena, Germany \\ E-mail: kog@fli-leibniz.de \\ Received August 9, 2010; revised September 14, 2010; accepted September 29, 2010
}

\begin{abstract}
The masses of a 11 fundamental elementary particles (those with a lifetime $>10^{-24} \mathrm{sec}$ ) can be calculated with an inaccuracy of approx. $1 \%$ using the equation $\mathrm{m} / \mathrm{m}_{\text {electron }}=\mathrm{N} / 2 \alpha$ where $\alpha$ is the coupling constant of quantum electrodynamics (also known as fine structure constant) $(=1 / 137.036)$, and $\mathrm{N}$ is an integer variable. This is the by far most accurate and most comprehensive approach to calculate the particle masses.
\end{abstract}

Keywords: Particle Masses, Fine Structure Constant

\section{Introduction}

While theories of elementary particles are accurate in determining decay channels and finding particle-multiplets, calculation of particle masses is less successful. The reason is that the masses of the constituting quarks cannot be simply added up - binding energies need to be considered which often exceed quark masses considerably. A comprehensive theory on particle masses is not available. Occasionally, the mass of one given elementary particle is predicted by a theory, but an accuracy in the $10 \%$ regime is considered already as satisfying. Better accuracies are only achieved when a theory is restricted to subsets of the elementary particles, for example the octet of mesons (the two pions and the four kaons) or the decuplet of baryons (delta, sigma xi, omega). Very good mass predictions have been obtained for the proton and its excited states. A quark model with one-gluonexchange gives an accuracy of $5.6 \%$ [1], an alternative model using "instantons" yields 5.1\% [2] and, as the best, the AdS/QCD model using di-quarks has an accuracy of $2.5 \%$ [3]. For an overview on these theories in German language see [4].

In contrast to those approaches, the present contribution aims at finding a simple rule which allows to give the masses of a 11 fundamental elementary particles with reasonable accuracy. A first attempt had been made earlier, however with errors of several percent particularly for the light particles [5].

\section{Basic Data}

There are 36 elementary particles with lifetimes longer than $10^{-24} \mathrm{sec}$. All others have shorter lifetime which are usually determined not by direct time measurements but by determining their line width. A lifetime of less than $10^{-24} \mathrm{sec}$ means that such a short living particle, even when moving with a speed close to that of light, cannot migrate even one own diameter before it decays. Table 1 gives the masses of all "fundamental" elementary particles:

All leptons have negative charge. For mesons with different charge, both charged mesons have the same mass. For baryons the following applies: $\mathrm{m}^{\text {positive }}<\mathrm{m}^{\text {neutral }}<$ $\mathrm{m}^{\text {negative }}$ (except for the double positive sigma $\mathrm{c}_{\mathrm{c}}$ ). Generally, the masses of the differently charged versions of a particle differ by less than $1 \%$, except for the pion, where the difference is $3.5 \%$. In the following, the particle variant with the longest lifetime (printed bold in Table 1) is taken as representative, with the exception of the kaon, where the mass of $\mathrm{K}^{+/}$(lifetime $1.238 \times 10^{-8} \mathrm{sec}$ ) is used instead of the mass of $\mathrm{K}_{\mathrm{L}}^{0}$ (slightly higher lifetime $5.18 \times$ $10^{-8} \mathrm{sec}$ ). The reason for using the mass of a variant with longest lifetime is that the present approach is not yet accurate enough to distinguish between charged and non charged versions of a particle. 
Table 1. Mass in $M e V / c^{2}$ for all fundamental elementary particles (modified from [6]). The particle variant with the longest lifetime is printed in bold.

\begin{tabular}{|c|c|c|c|}
\hline Particle & charged & neutral & \\
\hline \multicolumn{4}{|l|}{ LEPTONS } \\
\hline Electron & $0.511(-)$ & & \\
\hline Muon & $105.66(-)$ & & \\
\hline Tau & $1776.99(-)$ & & \\
\hline \multicolumn{4}{|l|}{ MESONS } \\
\hline Pion & $139.57(+/-)$ & 134.98 & \\
\hline Kaon & $493.68(+/-)$ & 497.65 & $\mathrm{Ks}^{0}$ and $\mathrm{K}_{\mathrm{L}}^{0}$ \\
\hline Eta & & 547.75 & \\
\hline Rho & & 775.8 & \\
\hline Omega & & 782.59 & \\
\hline D Meson & $1869.4(+/-)$ & & \\
\hline Ds Meson & $1968.3(+/-)$ & & \\
\hline B Meson & $5279.0(+/-)$ & 5279.4 & \\
\hline Bs Meson & & 5369.6 & \\
\hline BARYONS & positive & neutral & negative \\
\hline Nucleon & 938.27 & 939.56 & \\
\hline Lambda & & 1116.68 & \\
\hline Sigma & 1189.37 & 1192.64 & 1197.45 \\
\hline $\mathbf{X i}$ & & 1314.18 & 1321.31 \\
\hline Omega & & & 1672.45 \\
\hline Lmabda $_{c}$ & 2284.9 & & \\
\hline $\operatorname{Sigma}_{c}$ & $\begin{array}{c}(+) 2451.3 \\
(++) 2452.5\end{array}$ & 2452.2 & \\
\hline $\mathbf{X} \mathbf{i}_{\mathbf{c}}$ & 2466.3 & 2471.8 & \\
\hline Omega $_{c}$ & & 2697.5 & \\
\hline $\operatorname{Lambda}_{\mathrm{s}}^{0}$ & & 5624.0 & \\
\hline
\end{tabular}

\section{Results}

\section{1. $\alpha$ Governs Particle Masses}

The mass of the muon is $105.66 \mathrm{MeV} / \mathrm{c}^{2}$ or 206.77 times the electron mass which is approx $1.5 / \alpha$ electron masses, with an error of $0,59 \%$ as a result of $\alpha=1 / 137.036$. Correspondingly, the mass of the charged pions is $2 / \alpha$ electron masses and that of the kaons is $7 / \alpha$, both again with better than $1 \%$ inaccuracy. Thus, the masses of these particles can be expressed as the following:

$$
\mathrm{m} / \mathrm{m}_{\text {electron }}=\mathrm{N} / 2 \alpha
$$

with $\mathrm{N}=3$ for the muon, $\mathrm{N}=4$ for the pion and $\mathrm{N}=14$ for the kaon. This simple relationship suggests that the masses of other particles may also be expressed in such simple terms. The results are listed in Table 2.

In fact, the masses of a 11 fundamental elementary particles follow Equation (1) with a 1\% inaccuracy except for eta and omega mesons $(2.22 \%$ and $1.6 \%$, respectively), and the xi baryon (1.23\%). Figure 1 gives a graphical representation of the calculated particle mass versus the running number $\mathrm{N}$.

Table 2. Comparison of experimental and calculated particle masses, with the corresponding value of $\mathbf{N}$ and the difference in percent.

\begin{tabular}{|c|c|c|c|c|}
\hline Particle & $\exp$ & calc & $\mathrm{N}$ & $\%$ \\
\hline \multicolumn{5}{|l|}{ LEPTONS } \\
\hline Electron & 0.511 & --------- & & \\
\hline Muon & 105.66 & 105.04 & 3 & -0.59 \\
\hline Tau & 1776.99 & 1785.66 & 51 & 0.49 \\
\hline \multicolumn{5}{|l|}{ MESONS } \\
\hline Pion & 139.57 & 140.05 & 4 & 0.34 \\
\hline Kaon & 493.68 & 490.18 & 14 & -0.71 \\
\hline Eta & 547.75 & 560.21 & 16 & 2.22 \\
\hline Rho & 775.8 & 770.28 & 22 & -0.72 \\
\hline Omega & 782.59 & 770.28 & 22 & -1.60 \\
\hline D Meson & 1869.4 & 1855.69 & 53 & -0.74 \\
\hline Ds Meson & 1968.3 & 1960.73 & 56 & -0.39 \\
\hline B Meson & 5279.4 & 5251.94 & 150 & -0.52 \\
\hline Bs Meson & 5369.6 & 5391.99 & 154 & 0.42 \\
\hline \multicolumn{5}{|l|}{ BARYONS } \\
\hline Nucleon & 938.27 & 945.35 & 27 & 0.75 \\
\hline Lambda & 1115.68 & 1120.40 & 32 & 0.42 \\
\hline Sigma & 1197.45 & 1190.44 & 34 & -0.59 \\
\hline $\mathrm{Xi}$ & 1314.18 & 1330.49 & 38 & 1.23 \\
\hline Omega & 1672.45 & 1680.62 & 48 & 0.49 \\
\hline Lambda $_{c}$ & 2284.9 & 2275.84 & 65 & -0.40 \\
\hline Sigma $_{c}$ & 2452.2 & 2450.9 & 70 & -0.05 \\
\hline$X i_{c}$ & 2466.3 & 2450.9 & 70 & -0.63 \\
\hline Omega $_{c}$ & 2697.5 & 2696.0 & 77 & -0.06 \\
\hline $\operatorname{Lambda}^{0}{ }_{\mathrm{s}}$ & 5654.0 & 5637.09 & 161 & 0.23 \\
\hline
\end{tabular}




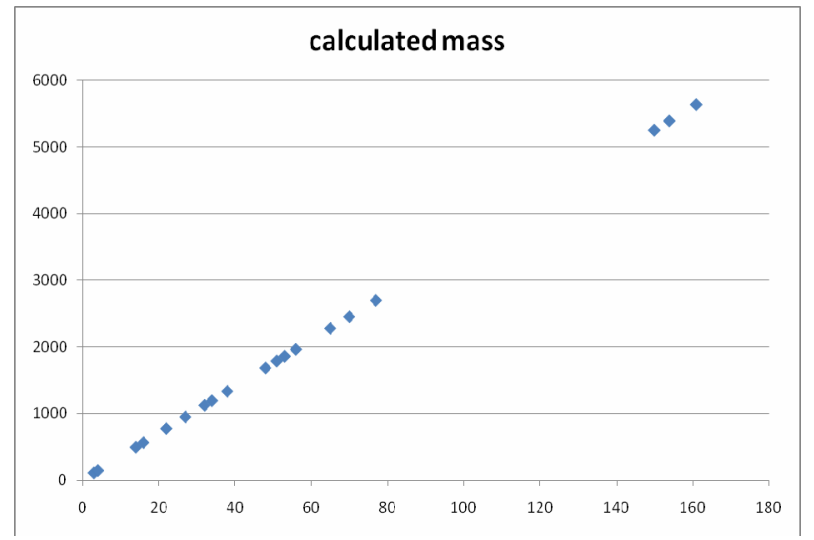

Figure 1. Particle mass as function of running number $\mathbf{N}$.

\subsection{Can This Result have been Obtained Simply by Chance?}

Since N in Equation (1) is a freely adaptable parameter, a good result may be simply achieved by chance, even when the choice of $\mathrm{N}$ is restricted to integer numbers. This is particularly the case for heavy particles. For example, with an $\mathrm{N}$ of $159,160,161,162$ the mass of lambda $_{b}$ (experimental value 5624) is calculated to be $5567,5602,5637,5672$, i.e. the fit is accurate within $1 \%$. Thus, an accurate calculation via Equation (1) of heavy masses is trivial. In the case of the light particles, for example the muon, using $\mathrm{N}=2$ or $\mathrm{N}=4$ would result in $33 \%$ difference instead of $0.6 \%$ for the obviously correct $\mathrm{N}=3$. For the heavier kaon, this error would still be an order of magnitude higher than given in Table 2. Therefore, even if the mass of a particle calculated separately for one isolated single particle would have been accurate simply by chance, the probability that this would be the case for the whole ensemble of all fundamental elementary particles is close to zero.

\section{Discussion}

Apart from the unprecedented accuracy of predicted masses, Equation (1) covers the whole ensemble of known elementary particles with lifetimes $>10^{-24} \mathrm{sec}$. This is distinctly more than other calculations of such masses, which typically are applied only for one or at most a few particles, and often do need more than one adaptable parameter. Intriguingly leptons, mesons and baryons equally well fit into this scheme. In the case of the nucleon Equation (1) can be used to calculate the fundamental constant of nature, $\beta=\mathrm{m}_{\text {proton }} / \mathrm{m}_{\text {electron }}$ with an accuracy of $0.8 \%$. To find out why only at the values of $\mathrm{N}$ listed in Table 2 "stable" particles are found, has to be the next step for understanding the nature of mass. Indeed, when also resonances with lifetimes smaller than $10^{-24} \mathrm{sec}$ are included, most "empty" values of $\mathrm{N}$ can be filled [5], however then with lower accuracy for the whole ensemble of particles and resonances.

The results of the present work have to be seen in the context of ref. [7], where the masses of a number of selected particles have been calculated with even better accuracy. For example, the mass of the uncharged pion is there calculated to be $139.96 \mathrm{MeV} / \mathrm{c}^{2}$ and that of the charged version $134,97 \mathrm{MeV} / \mathrm{c}^{2}$, i.e. exact and distinguishing between the different variants of the particle. The disadvantage of that approach is, that a large number of parameters is needed and that two basic masses, one with $29.7918 \mathrm{MeV} / \mathrm{c}^{2}$ and one with $26,1299 \mathrm{Mev} / \mathrm{c}^{2}$ are used. In contrast, in the present consideration, the ensemble of a 11 particles can be uniquely represented by multiples of $35,01 \mathrm{MeV} / \mathrm{c}^{2}$. Though a strict physical explanation is still elusive, formally this is, within the large error bars of quark masses, the average of the masses of the up- ,down- and strange quark $\left(37,7+/-8 \mathrm{MeV} / \mathrm{c}^{2}\right)$.

The fact that a very simple equation, solely based on $\alpha$, a basic constant of quantum electrodynamics and spectroscopy, predicts particle masses with high accuracy indicates that the result presented in this work has indeed a real physical background. In a further step, such insights will have to be correlated to theories on the Higgs particle as mediator of mass.

\section{References}

[1] S. Capstick and N. Isgur, "Baryons in a Relativized Quark Model with Chromodynamics," Physical Review D, Vol. 34, No. 9, 1986, pp. 2809-2835.

[2] U. Löring, B. C. Metsch and H. R. Petry, "The LightBaryon Spectrum in a Relativistic Quark Model with Instanton-Induced Quark Forces," European Physical Journal A, Vol. 10, No. 4, April 2001, pp. 395-446.

[3] H. Forkel and E. Klempt, "Diquark Correlations in Baryon Spectroscopy and Holographic Qcd," Physics Letters B, Vol. 679, No. 1, August 2009, pp. 77-80.

[4] E. Klempt, "Vom Ursprung der Masse (On the origin of mass)," Physik Journal, Vol. 9, 2010, pp. 31-37.

[5] K. O. Greulich, "An Empirical One-Parameter Equation for Elementary Particle Masses," Verhandlungen der Deutschen Physikalischen Gesellschaft (Proceedings of the German Physical Society), March 2007, Gr 603.

[6] K. Bethge and U. Schröder, "Elementarteilchen und ihre Wechselwirkungen (Elementary particles and their interactions)," Wiley VCH, Weinheim, 2006.

[7] A. G. Kyriakos, "On Calculation of Elementary Particles' Masses," arxiv.org/pdf/physics/0604063, 2006. 